(1985) Pferdeheilkunde 1, 99-108

\section{Röntgenologie des Kniegelenks beim Pferd}

\author{
L. B. Jeffcott
}

Department of Veterinary Clinical Sciences, University of Melbourne,

Australia

\section{Röntgentechnik}

Es hat klare Vorzüge, Röntgenaufnahmen des Kniegelenks unter Allgemeinnarkose auszuführen, als da sind bessere Standardisierung der Belichtungsfaktoren, reduzierte Unschärfe durch Bewegung, ein größerer Spielraum möglicher Projektionen und viel höhere Strahlensicherheit. In der Praxis wird man diese Faktoren gegenüber den Nachteilen und potentiellen Risiken der Narkose abzuwägen haben.

Ein Ausstattungsteil, das am stehenden und am liegenden Tier benutzt werden kann, um die Bildqualität zu verbessern, ist das Dodger-T Aluminium-Filter (Saab-Scania, Linkoping, Schweden). Es vermindert die Primärstrahlung durch das Weichteilgewebe und dünnere Strukturen und reduziert das Ausmaß der Streustrahlung. (Jeffcott, 1979).

Es ist ratsam, Röntgenaufnahmen immer in mehreren Ebenen anzufertigen, und dabei ist die aufschlußreichste Ansicht die lateromediale (LM) und die kaudokraniale (postoanteriore, PA) (Jeffcott und Kold, 1982a). Am stehenden Pferd ist ein wertvoller praktischer Tip für die LM-Ansicht, die Gliedmaße anzuheben und nach kaudal zu strekken. Dies führt zu einer leichten Beugung und ventralen Verlagerung des Kniegelenks, wodurch die Kassette besser angelegt werden kann, eine genauere LM-Projektion des Gelenks in einer Linie erreicht und eine reduzierte Belichtung benötigt wird.

\section{Röntgenanatomie und Zufallsbefunde}

Das Pferdeknie ist das größte Gelenk im Körper und ein komplexes anatomisches Gebilde; daher ist eine detaillierte Kenntnis der röntgenanatomischen Verhältnisse entscheidend.

Bei der Geburt ist die Ossifikation unvollständig und das röntgenologische Erscheinungsbild weicht stark von dem des ausgewachsenen Pferdes ab.

Die Epiphysenfugen distal am Femur und proximal an der Tibia treten sehr deutlich hervor und verlaufen an den Rändern gewöhnlich nach oben (Abb. 1a und 1b).

Der Gelenkspalt des Femorotibialgelenks erscheint viel weiter als beim erwachsenen Pferd, und die Fossa intercondylaris ist sehr flach. Der äußere Rand des medialen Kondylus kann konvex statt konkav sein. Der Zwischenknorrenfortsatz ist nicht voll ausgebildet, und die Ossifikation der Fibula ist im allgemeinen noch sehr gering. Beim neu-
Tab. 1: Zeitpunkte des Auftretens von Ossifikationskernen am Kniegelenk sowie des Fugenschlusses und der Beendigung des Wachstums

\begin{tabular}{llll}
\hline & Stelle & $\begin{array}{l}\text { Zeitpunkt des } \\
\text { Auftretens } \\
\text { (Trächtigkeits- } \\
\text { dauer in Tagen) }\end{array}$ & $\begin{array}{l}\text { Zeitpunkt des } \\
\text { Schlusses } \\
\text { (Alter in } \\
\text { Monaten) }\end{array}$ \\
\hline Femur & $\begin{array}{l}\text { Diaphyse } \\
\text { distale Epiph. }\end{array}$ & $\begin{array}{c}60-70 \\
220-145\end{array}$ & $21-42$ \\
\hline Tibia & $\begin{array}{l}\text { Prox. Epiphyse } \\
\text { Tuberos. tibiae }\end{array}$ & $\begin{array}{l}265-300 \\
290-320\end{array}$ & $36-42$ \\
& Diaphyse & $60-70$ & \\
\hline Fibula & $\begin{array}{l}\text { Prox. Epiphyse } \\
\text { und Diaphyse }\end{array}$ & nach der Geburt & 40 \\
\hline
\end{tabular}

geborenen Fohlen tritt die markante Diskrepanz zwischen dem medialen und lateralen Rollkamm der Tibia auf dem Röntgenbild nicht in Erscheinung, und die Patella hat einen einfachen, fast dreieckigen Umriß.

Im kranialen Bereich der proximalen Abrundung der Trochlea femoris und der Patella besteht eine auffällige Rauhigkeit und Unregelmäßigkeit.

Diese Ausprägungsformen verschwinden schrittweise, wenn der Ossifikationskern die Gestalt seines knorpeligen Vorläufers annimmt (in der Regel im Alter von zwei bis drei Monaten).

Die distale Femurepiphysenfuge wird im Lauf der Entwicklung in ihrer Kontur etwas wellig und unregelmäßig (Abb. 2). Im kaudalen Bereich zeigt sie oft in der Mitte Bereiche früher Sklerosierung und periostale Aufrauhungen. Der Fugenschluß tritt hier und an der proximalen Tibiaepiphyse etwa vom 20. Lebensmonat an ein (Tab. 1). Die Tuberositas tibiae besitzt ein völlig separates Ossifikationszentrum, das endgültig nicht vor Erreichen eines Alters von neun bis zwölf Monaten mit dem Tibiaschaft verschmilzt. Die Fibula beginnt vom Kern an ihrem Köpfchen aus zu verknöchern, was im Alter von 2 Monaten deutlich sichtbar ist, und behält eine persistierende Knorpellinie, die als Fibulafraktur fehldiagnostiziert werden kann (Abb. 3).

Die Umrisse der beiden Trochlearollkämme erscheinen nach den ersten Lebenswochen sehr verschieden voneinander. Der mediale Rollkamm ist viel größer, besonders proximal, wo er breiter und stärker gerundet ist. Gewöhnlich besteht am distalen Ende der Trochlea da, wo der mediale Kondylus entsteht, eine kleine knotenförmige Verschattung. Auf der lateralen Seite ist an dieser Stelle am Übergang ein abgeflachter und leicht aufgerauhter Bezirk vorhanden (Abb. 4a und 4b). In der PA-Projektion ist die Kontur des medialen Epikondylus als relativ glatte konkave Oberfläche zu sehen. Der Umriß des lateralen Epikondylus tritt mehr konvex hervor und erscheint oft ziemlich unregelmäßig und rauh. Beim erwachsenen Pferd zeigt die kraniale Fläche der Patella oft Unregelmäßigkeiten und Rauhigkeiten, die nicht mit röntgenologischer Manifestation einer Überbelastung des Ligamentum patellae verwechselt werden sollten. Es gibt relativ wenig Zufallsbefunde, die die röntgenologische Beurteilung der Kniegelenksregion erschweren. Eine Zusammenstellung davon mit Differentialdiagnosen zeigt Tabelle 2 . 

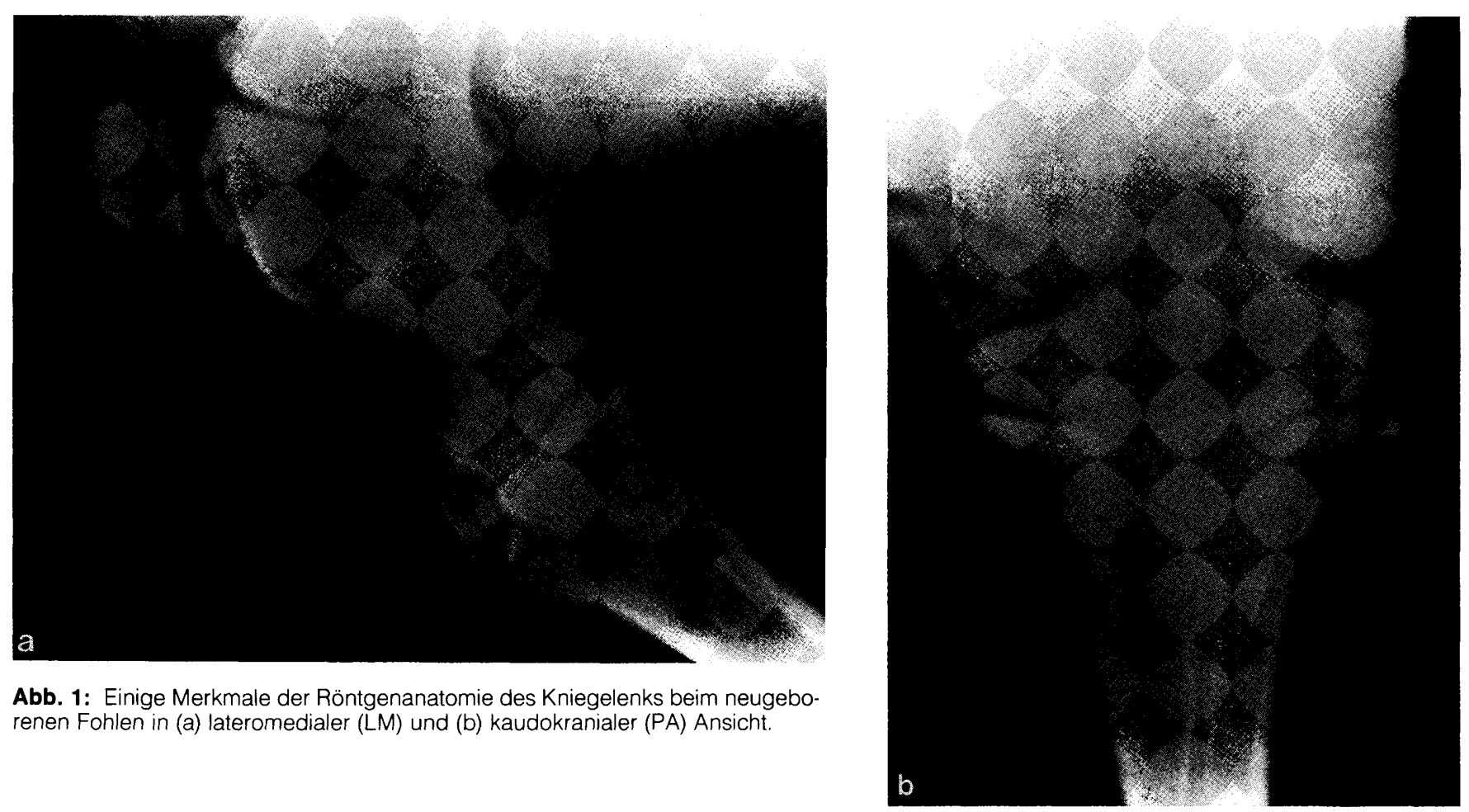

Abb. 1: Einige Merkmale der Röntgenanatomie des Kniegelenks beim neugeborenen Fohlen in (a) lateromedialer (LM) und (b) kaudokranialer (PA) Ansicht.

\section{Röntgenbefunde}

Es gibt einen weiten Bereich von Röntgenbefunden, die am Kniegelenk erhoben werden können, und Tabelle 3 stellt eine Auflistung der wichtigeren dar.

\section{Frakturen}

Schwere Frakturen und Splitterbrüche im Kniegelenksbereich verursachen gewöhnlich keinerlei diagnostische Schwierigkeiten. In den meisten Fällen sind davon der distale Femurschaft und die Patella betroffen. Die erkrankte Gliedmaße wird oft halb gebeugt gehalten, so daß das Kniegelenk tief und nach vorne hängt, weil das Tier die Gliedmaße kaum mehr in nennenswertem Maß belasten kann. Unter diesen Umständen können befriedigende Röntgenaufnahmen im Stehen so angefertigt werden, daß es für den Patienten möglichst wenig unangenehm ist, indem das Röntgenstrahlenbündel von der gesunden Seite her unter dem Abdomen hindurch dirigiert wird und man so eine mediolaterale Ansicht erhält.

\section{Osteochondrose}

Die Osteochondrosis dissecans kann das Kniegelenk besonders bei jungen Tieren im Alter zwischen zwei Monaten und zwei Jahren betreffen. Allgemein ist der am Röntgenbild erhobene Befund desto gravierender und die Prognose desto ungünstiger, je früher klinische Erscheinungen auftreten. Röntgenologisch tendieren die Läsionen dazu, auf das Femoropatellargelenk beschränkt zu bleiben. Hauptsächlich betroffen ist der laterale Rollkamm der Trochlea, aber der mediale Rollkamm und die distale Gelenkfläche der Patella können ebenfalls verändert sein. Es bestehen deutliche Unregelmäßigkeiten der Gelenkkontur mit flekkigen Aufhellungszonen und lokaler Sklerosierung infolge

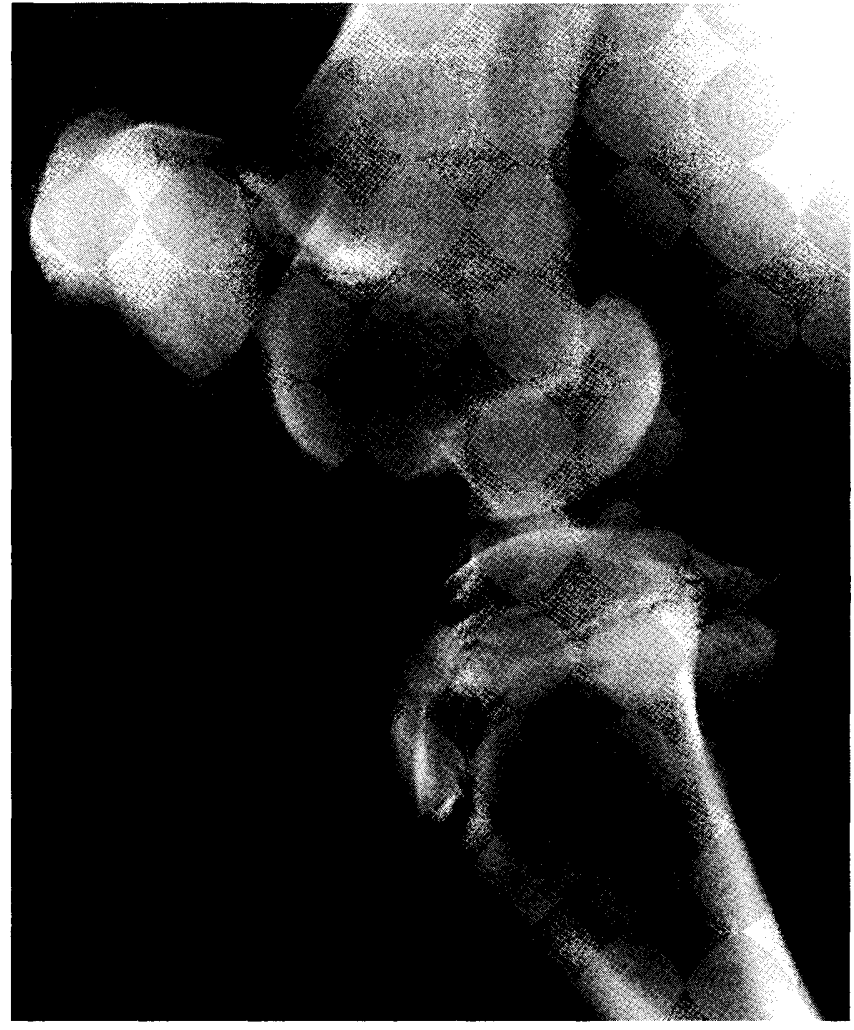

Abb. 2: Röntgenanatomie (LM-Ansicht) eines fünf Monate alten Fohlens.

des rupturierten Knorpels und kollabierten subchondralen Knochengewebes (Abb. 5a).

Wenn die Veränderungen nicht so schwer sind, treten klinische Erscheinungen oft erst auf, wenn das Tier in Arbeit oder Training genommen wird (d. h. mit zwei Jahren oder 


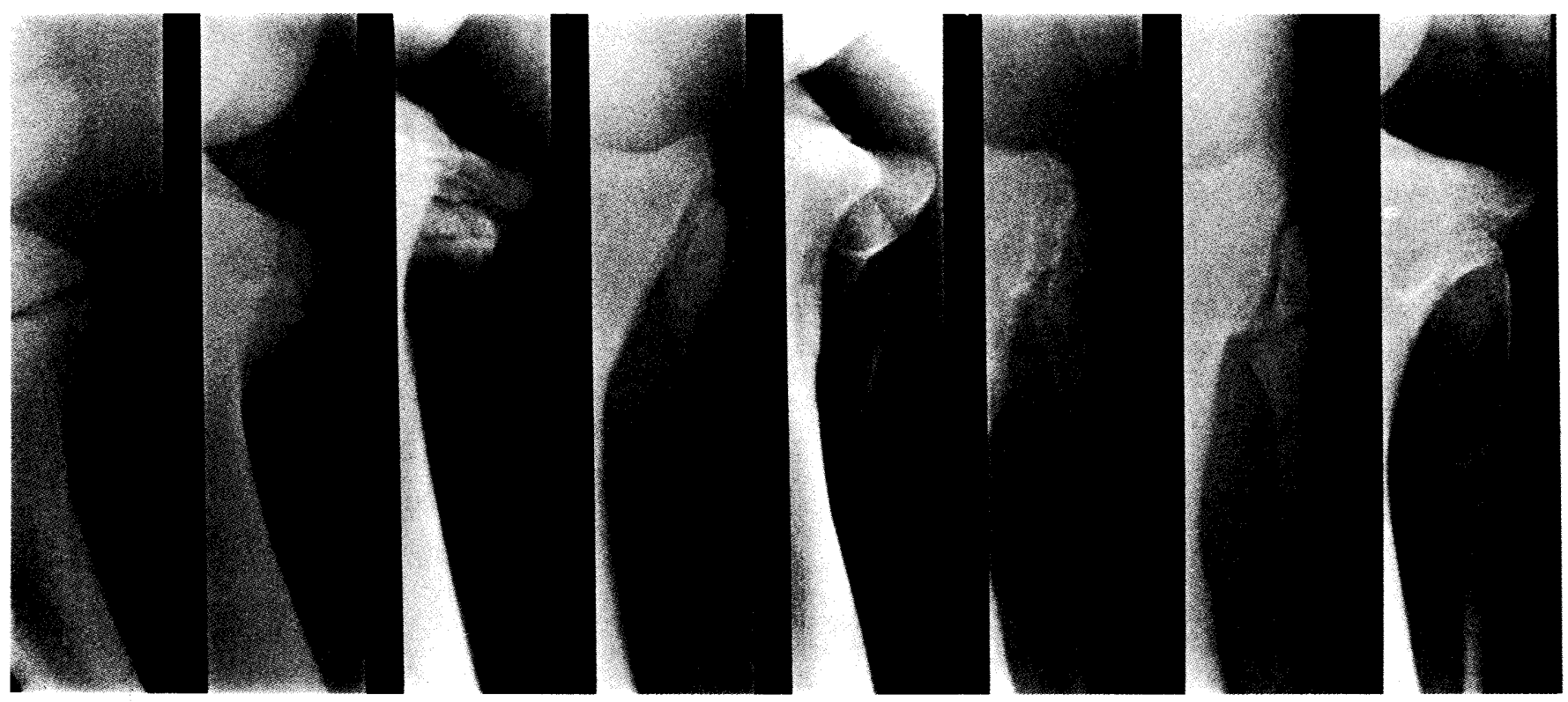

Abb. 3: Röntgenaufnahmen der Fibula des Pferdes als Beispiele für den Ossifikationsprozeß, unvollständige Ossifikation und Fraktur.

später). Die röntgenologisch sichtbaren Läsionen sind mehr lokalisiert und umschrieben, betreffen aber wieder hauptsächlich den kranialen Mittelteil des lateralen Rollkamms der Tibia (Abb. 5b). In vielen Fällen ist eine ossifizierte "Gelenkmaus" vorhanden, die sich frei im Femoropatellargelenk bewegt oder noch in Verbindung zum Roll-

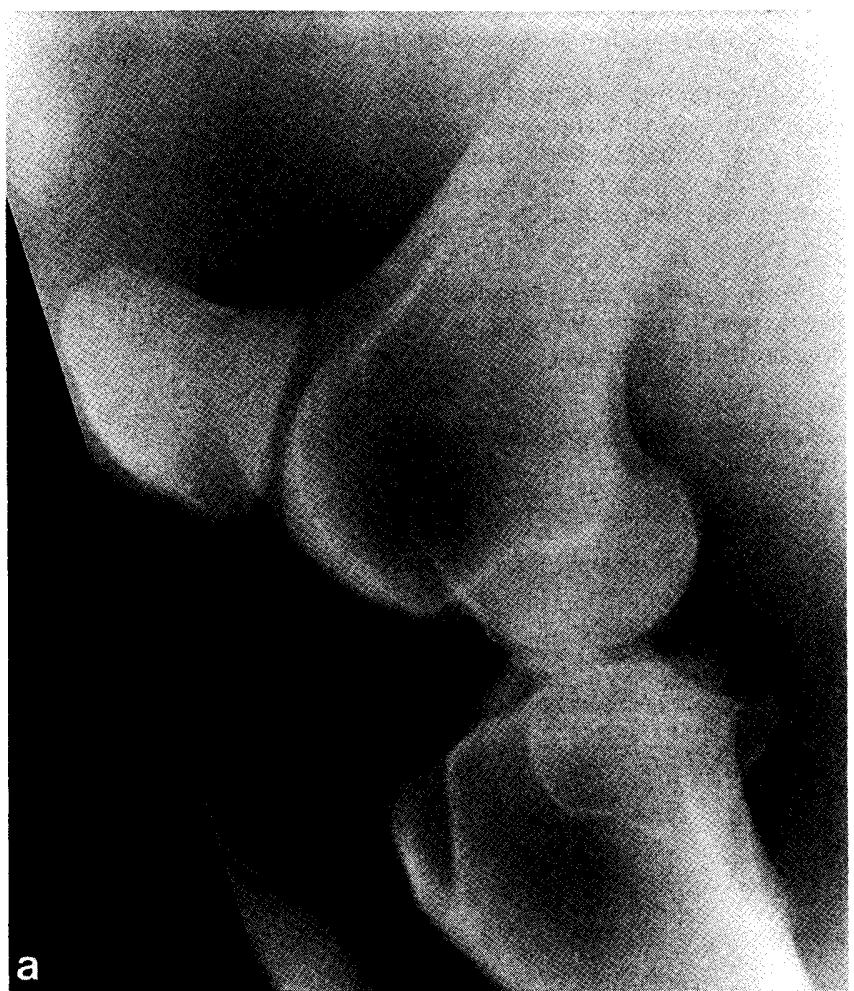

kamm der Trochlea steht. Eine Abflachung des Trochlearollkamms ist in länger bestehenden Fällen zu beobachten. Die Prognose ist in diesen Fällen von Osteochondrosis dissecans günstiger, und einige Pferde sprechen auf eine chirurgische Behandlung mit Kürettage des degenerierten Gelenkknorpels an.

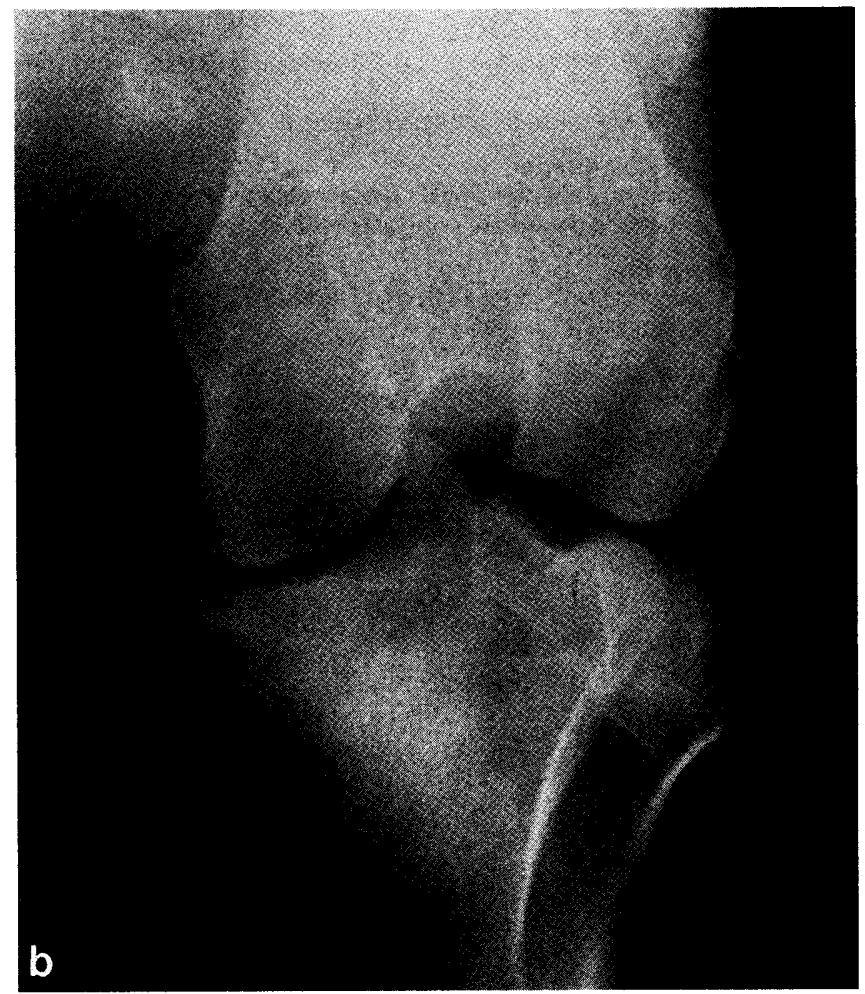

Abb. 4: Röntgenanatomie des Kniegelenks beim ausgewachsenen Pferd, (a) LM- und (b) PA-Ansicht. 


\begin{tabular}{|c|c|}
\hline Zufallsbefund & Differentialdiagnose \\
\hline $\begin{array}{l}\text { Deutlich hervortretende wellige Linie der } \\
\text { distalen Femurepiphyse, manchmal ver- } \\
\text { bunden mit leichter Sklerose, im Alter von } \\
6 \text { bis } 12 \text { Monaten }\end{array}$ & Epiphysitis \\
\hline $\begin{array}{l}\text { Vor dem } 12 \text {. Lebensmonat besteht ein voll- } \\
\text { ständig oder teilweise separater Ossifika- } \\
\text { tionskern der Tuberositas tibiae }\end{array}$ & $\begin{array}{l}\text { proximale } \\
\text { Tibiafraktur }\end{array}$ \\
\hline $\begin{array}{l}\text { Unvollständige Verknöcherung der Fibula } \\
\text { in jedem Lebensalter }\end{array}$ & Fibulafraktur \\
\hline $\begin{array}{l}\text { Abflachung der Trochlea am distalen Ende } \\
\text { des Femoropatellargelenks }\end{array}$ & Osteochondrose \\
\hline $\begin{array}{l}\text { Aufrauhung in der kranialen Ansicht der } \\
\text { Patella }\end{array}$ & $\begin{array}{l}\text { Überbelastung } \\
\text { des geraden } \\
\text { Kniescheibenbands }\end{array}$ \\
\hline $\begin{array}{l}\text { In der PA-Projektion scheint die Patella, } \\
\text { wenn das Tier die Gliedmaße belastet, } \\
\text { über oder etwas lateral vom lateralen Roll- } \\
\text { kamm der Trochlea zu liegen }\end{array}$ & Patella-Luxation \\
\hline $\begin{array}{l}\text { Aufrauhungen und Unregelmäßigkeiten auf } \\
\text { der lateralen Oberfläche des lateralen Kon- } \\
\text { dylus }\end{array}$ & $\begin{array}{l}\text { Überbelastung } \\
\text { des lateralen } \\
\text { Kollateralbands }\end{array}$ \\
\hline $\begin{array}{l}\text { Aufhellung oben lateral an der Fossa inter- } \\
\text { condylaris und neben dem Zwischenknor- } \\
\text { renfortsatz der Tibia in der proximalen Ti- } \\
\text { biaepiphyse in PA-Ansicht }\end{array}$ & $\begin{array}{l}\text { subchondrale } \\
\text { Knochenzyste }\end{array}$ \\
\hline $\begin{array}{l}\text { Aufrauhungen und Unregelmäßigkeiten in } \\
\text { der kranialen proximalen Ansicht der Patel- } \\
\text { la und der Trochlae bei jungen Fohlen }\end{array}$ & eitrige Arthritis \\
\hline $\begin{array}{l}\text { Frühe dornförmige Ausziehung am media- } \\
\text { len Kondylus der Tibia, die nicht in Bezie- } \\
\text { hung zum Femorotibialgelenk steht }\end{array}$ & $\begin{array}{l}\text { sekundäre Gelenks- } \\
\text { degeneration }\end{array}$ \\
\hline $\begin{array}{l}\text { Scheinbar kollabierter Gelenkspalt des Fe- } \\
\text { morotibialgelenks, hervorgerufen durch un- } \\
\text { geeignete Röntgentechnik }\end{array}$ & $\begin{array}{l}\text { Meniskusverletzung } \\
\text { oder sekundäre } \\
\text { Osteoarthritis }\end{array}$ \\
\hline
\end{tabular}

Tab. 2: Röntgenologische Zufallsbefunde im Kniegelenksbereich des Pferdes

\section{Subchondrale Knochenzysten}

Das Vorhandensein großer zystischer Läsionen am Kniegelenk des Pferdes stellt eine wichtige Lahmheitsursache besonders bei jungen Tieren dar. Es treten zwei Arten pathologischer Veränderungen auf, die röntgenologisch leicht $\mathrm{zu}$ unterscheiden sind. Der am weitesten verbreitete Typ von Zyste (Gruppe A) erscheint als einzelne klar begrenzte Aufhellungszone, $1-2 \mathrm{~cm}$ im Durchmesser, in der Mitte des medialen Femurkondylus in unmittelbarer Nachbarschaft

Tab. 3: Röntgenbefunde am Kniegelenk des Pferdes

\begin{tabular}{ll}
\hline $\begin{array}{l}\text { Fraktur } \\
\text { Femur - distaler Schaft } \\
\text { Osteochondral }\end{array}$ & $\begin{array}{l}\text { Epiphysitis } \\
\text { distale Femurepiphyse }\end{array}$ \\
Trochleafrakturen & $\begin{array}{l}\text { Andere Gelenkläsionen } \\
\text { Osteoarthritis }\end{array}$ \\
Tibia - proximale Epiphyse & (Femorotibialgelenk) \\
$\quad$ und Tuberositas & Eitrige Arthritis \\
Zwischenknorrentortsatz & (Fohlenlähme) \\
Fibula - Schaft & Meniskusverletzung \\
Osteochondrosis dissecans & Bänderriß \\
Rollkämme der Trochlea & gerades Kniescheibenband \\
Patella & kraniales Kreuzband \\
Subchondrale Knochenzysten & mediales Kollateralband \\
Medialer Femurkondylus (Gruppe A) & Verschiedenes \\
Fossa intercondylaris und & Laterale Patella-Luxation \\
proximale Tibia (Gruppe B) & Calcinosis circumscripta
\end{tabular}

des Femorotibialgelenks (Abb. 6a und 6b). Andere zystische Läsionen (Gruppe B) sind gelegentlich neben der Fossa intercondylaris und am proximalen Tibiaende direkt unter dem Zwischenknorrenfortsatz zu beobachten (Abb. 6c und $6 \mathrm{~d}$ ).

In Gruppe A ist die Lokalisation der Zysten bemerkenswert konstant. Nur sehr selten ist eine zweite kleinere $Z y$ ste („Kissing-type-Zyste“) an der gegenüberliegenden Gelenkfläche der proximalen Tibia sichtbar (Jeffcott und Kold, 1982c). In einigen Fällen (weniger als 20 Prozent) werden beiderseits Zysten gefunden, obwohl die Lahmheit einseitig ist. Die Form dieser Zysten kann rund, kuppenförmig oder oval sein. Das Zystenlumen kommuniziert immer mit dem Femorotibialgelenk, und eine sklerotische Zone weist oft hilfreich auf eine vorsichtig günstige Prognose hin. Zwar ossifizieren die Zysten nicht und verschwinden auch nicht mehr vollständig, aber eine gewisse Zunahme der Strahlendichte tritt im Lauf der Zeit oft ein.

Die Zysten der Gruppe B neigen dazu, einen unregelmäßigeren Umriß zu haben, und können in unterschiedlicher Größe und Zahl vorhanden sein (weniger als $2 \mathrm{~cm}$ im Durchmesser). Dies kann dem betroffenen Bereich des Femurkondylus oder der Tibia ein fast mottenzerfressenes Aussehen verleihen. Eine Verbindung zum Femorotibialgelenk ist nicht immer sichtbar, ebensowenig eine Sklerosierung in der Zystenperipherie.

Die Prognose bei Zysten der Gruppe A ist gewöhnlich recht günstig, besonders bei Tieren im Alter bis zu zwei Jahren, von denen über 60 Prozent die Gesundheit wiedererlangen. Bei solchen mit persistierender oder hochgradiger Lahmheit kann als eine Möglichkeit auf die chirurgische Behandlung zurückgegriffen werden, die in Kürettage der Zyste und Ausfüllung mit Knochenspänen besteht (Kold und Hickman, 1983). Viel geringer ist die Wahrscheinlichkeit der Genesung bei Pferden mit Zysten der Gruppe B, und sie können selten wieder voll gearbeitet werden.

Die Ätiologie dieser subchondralen Knochenzysten ist nicht genau bekannt, obwohl manche Autoren sie zum Osteochondrosekomplex rechnen (Strömberg, 1979). Es ist möglich, daß die Pathogenese der Zysten der Gruppe A durch zusätzliche andere Faktoren kompliziert wird wie fokussierte Gelenkschädigung infolge anormalen Drucks oder anormaler Belastung im Gelenk (Jeffcott, Kold und Melsen, 1983) oder einfach durch Traumen (Verschooten und De Moor, 1982). Die Zysten der Gruppe B können sich sekundär nach chronischer Schädigung der Ansatzstelle der Kreuzbänder proximal an der Tibia oder in der Fossa intercondylaris entwickeln. Man hofft, daß diese Fragen mit der Vervollständigung der laufenden Untersuchungen geklärt werden.

\section{Epiphysitis}

Am Kniegelenk ist im allgemeinen die Epiphysenfuge des distalen Femurs betroffen. Die Erkrankung tritt an dieser Stelle jedoch verglichen mit den Epiphysenfugen distal an Radius und Tibia sehr selten auf. Wenn es dazu kommt (Abb. 7), sind diese anderen, der Röntgenuntersuchung besser zugänglichen Stellen gleichermaßen befallen. Der Befund wird im Alter von 9 bis 12 Monaten klinisch manifest 


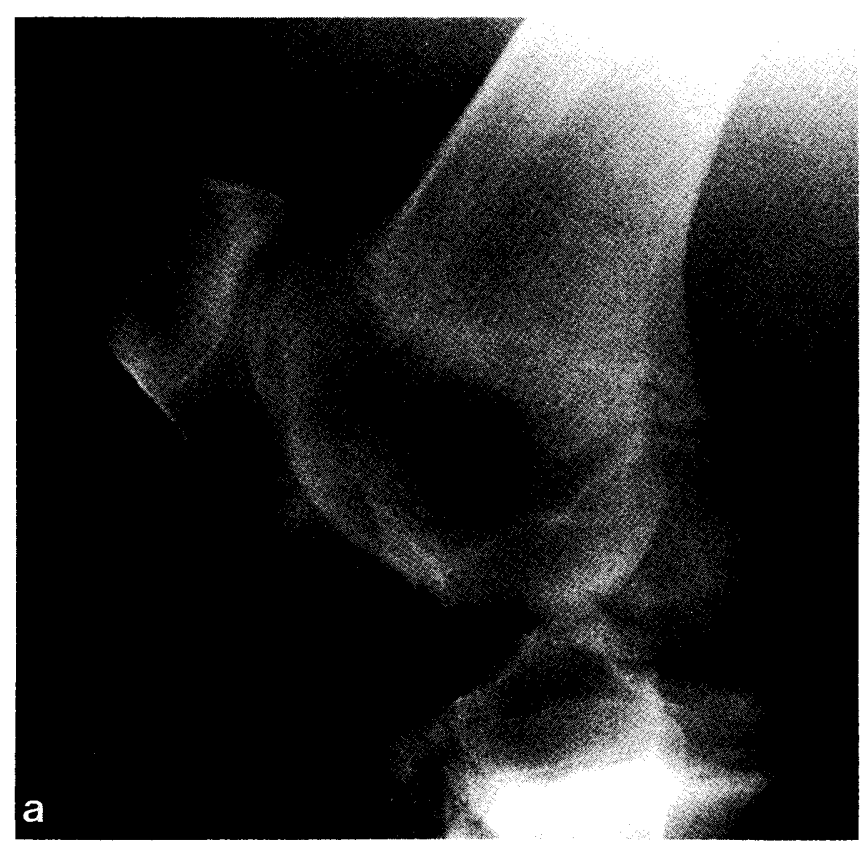

Abb. 5a: Ein Fall von schwerer Osteochondrosis dissecans, die den gesamten lateralen Rollkamm der Trochlea bei einem Fohlen befallen hat.

in Form von sehr steiler Stellung der Hintergliedmaßen, verkürzten steifen Schritten der Hinterhand und erworbener leichter bis hochgradiger Kyphose der Lendenwirbelsäule (Fischrücken). Normalerweise ist keine spezifische Behandlung erforderlich, allenfalls die Wachstumsgeschwindigkeit für einige Monate zu beschränken.

\section{Andere Gelenkläsionen}

Die Arthrosis deformans ist am Kniegelenk viel weniger weit verbreitet als an den Gelenken der distalen GliedmaBenabschnitte. Sie befällt hauptsächlich das Femorotibialgelenk älterer Tiere, die dann deutliche Muskelatrophie, chronische Steifheit und eine Lahmheit zeigen, die nicht auf antiinflammatorische Medikation anspricht. Die Er-
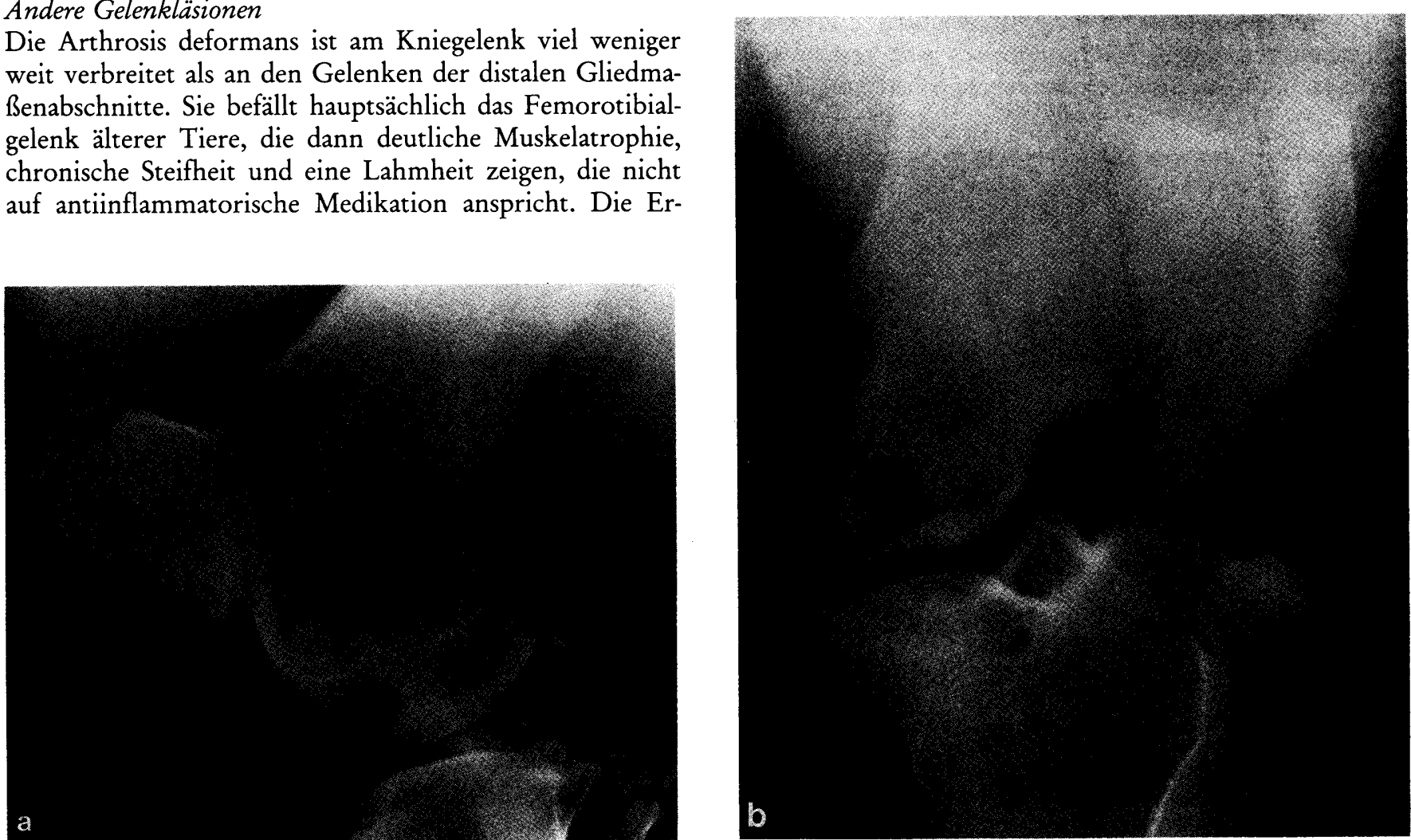

Abb. 6a und 6b: Große subchondrale Knochenzyste im medialen Femurkondylus (Gruppe A) in (a) LM- und (b) PA-Projektion. 


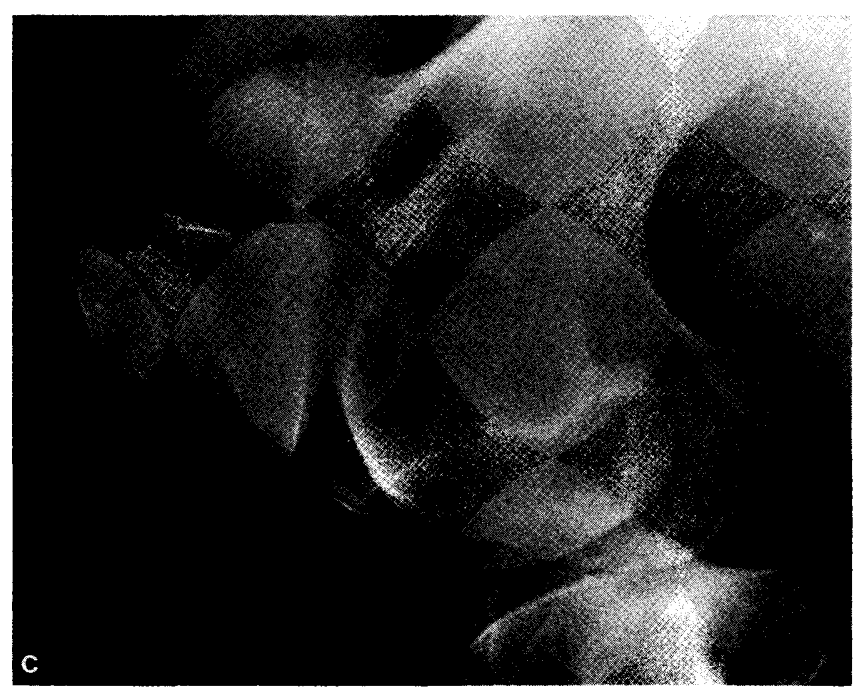

Abb. 6c und 6d: Zystische Degeneration, die Fossa intercondylaris umgebend (Gruppe B), in (c) LM- und (d) PA-Ansicht.

scheinungen auf dem Röntgenbild sind in der Regel weniger dramatisch als das klinische Bild. Wenn sich das Krankheitsgeschehen offensichtlich im Anfangsstadium befindet, ist der laterale Gelenksabschnitt schwerer betroffen, sichtbar als periartikuläre Knochenzubildung (Abb. 8). Der Zustand tritt auch sekundär nach anderen Ursachen chronischer Schädigung des Femorotibialgelenks (z. B. Kreuzband- oder Meniskusverletzung) auf; dann sieht man u. U. Anlagerung von Knochengewebe, Ausziehungen am Rand und Verschmälerung des Gelenkspalts.

Die eitrige Arthritis junger Fohlen (Fohlenlähme) ist klinisch mit vermehrter Wärme, Schwellung und akuter Lahmheit verbunden. Röntgenologisch sind keine Veränderungen sichtbar, bis eine ausgedehnte Schädigung der Ge-

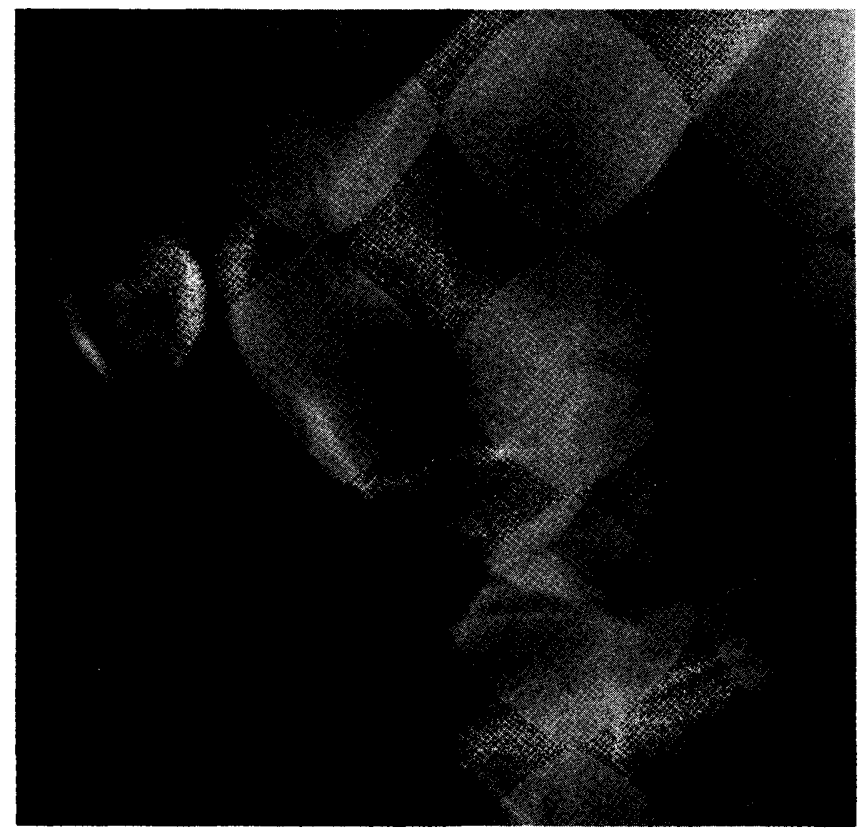

Abb. 7: Röntgenologische Veränderungen bei der Epiphysitis am distalen Femur eines 10 Monate alten Vollblutfohlens.

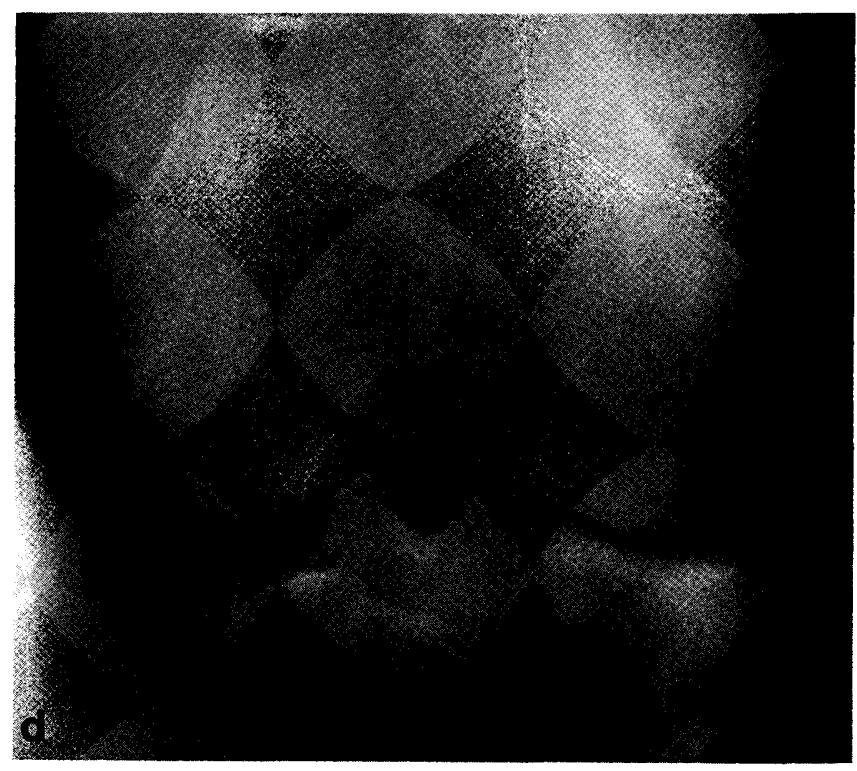

lenkflächen, des subchondralen Knochens oder der Epiphysenfuge stattgefunden hat (Dik, 1981). Diese Veränderungen sollten nicht mit den unregelmäßigen Konturen des Ossifikationskerns bei neugeborenen Fohlen verwechselt werden, und es müssen immer auch Röntgenaufnahmen der Gliedmaße gegenüber angefertigt werden, wenn die Erscheinungen einseitig auftreten. Röntgenologisch bedeutsame Läsionen sind anfangs als kleinere Defekte sichtbar, die

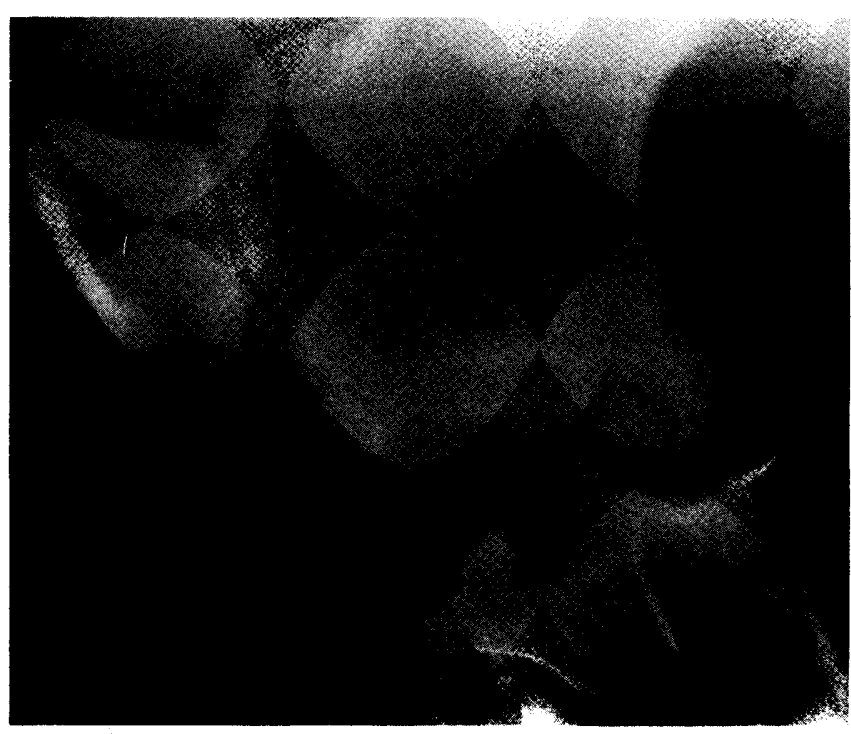

Abb. 8: Arthrosis deformans des Kniegelenks mit Veränderungen in Form von periartikulärer Knochenzubildung und dornförmiger Ausziehung.

sich zu deutlich aufgehellter Sequestration, Zusammenbruch und Zersplitterung des geschwächten Knochens weiterentwickeln. Diese Veränderungen können sehr rasch entstehen, benötigen aber mindestens eine Woche.

Die Meniskusverletzung geht mit chronischer Lahmheit und Umfangsvermehrung des Femorotibialgelenks einher. 

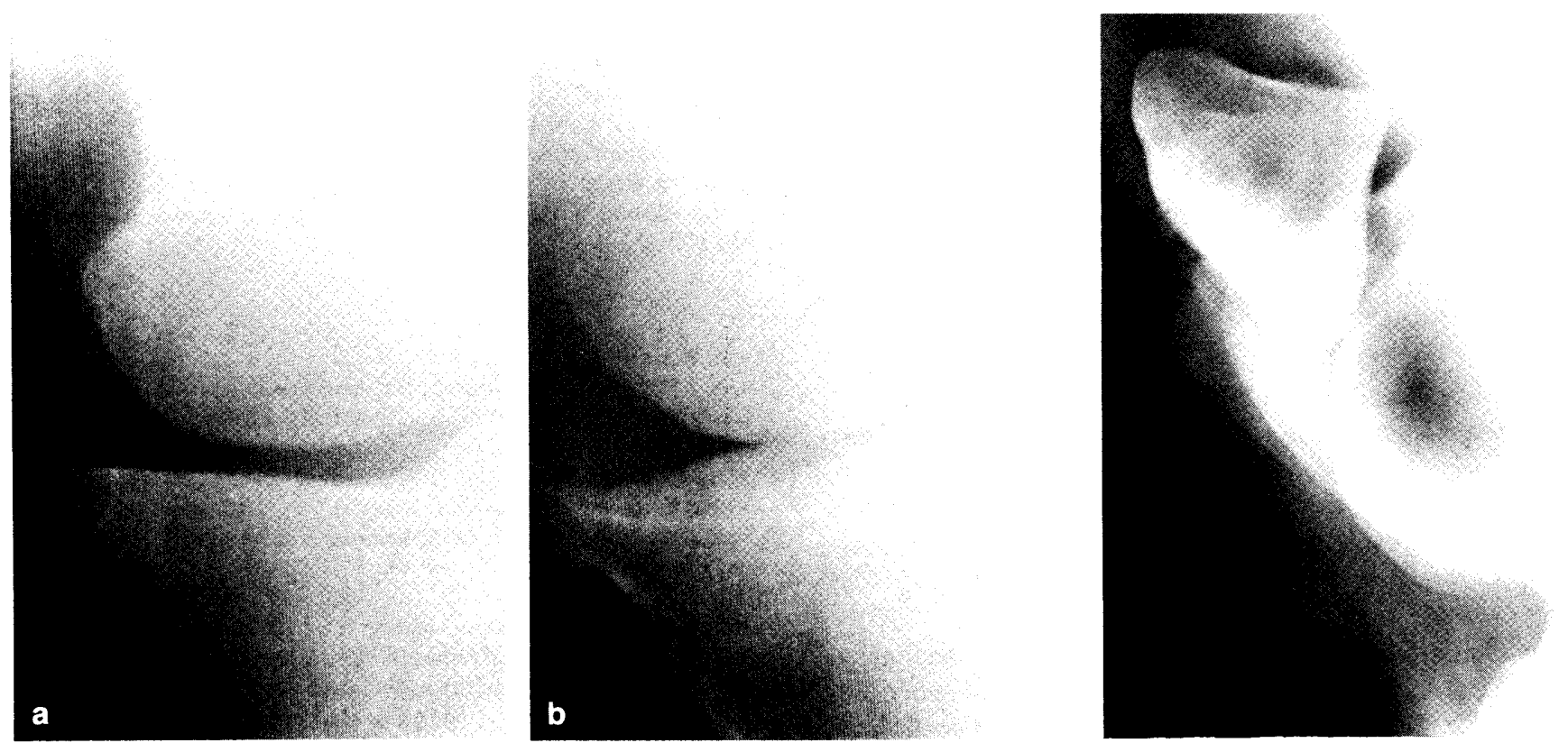

Abb. 9a und 9b: Meniskus-Verletzung, als kollabierter medialer Abschnitt des Femorotibialgelenks und dornförmige Ausziehung am Gelenkrand in Erscheinung tretend.

Abb. 10: Überbelastung des mittleren geraden Kniescheibenbands mit Weichteilschwellung über der Patella und Aufrauhung ihrer Kranialund Ventralfläche.

Auf den Röntgenaufnahmen besteht eine Verschmälerung des betroffenen Gelenkspalts, gewöhnlich des medialen. In vielen Fällen ist das mediale Kollateralband mehr oder weniger stark geschädigt, und man sieht dies in länger bestehenden Fällen als Aufrauhung an den Ansatzstellen des Bandes sowie randständigen Ausziehungen des Femorotibialgelenks (Abb. 9). Dies ist ein Fall, in dem die Röntgenuntersuchung im Stehen (d. h. unter Belastung) durchgeführt werden muß, wenn der Gelenkspalt beurteilt werden soll. Man trifft Meniskusverletzungen im United Kingdom und in Europa kaum an, sieht sie aber in größerer Verbreitung in den USA. Die Prognose bei diesen Pferden ist ungünstig, weil sich sekundär Degeneration und als Folge Instabilität des Femorotibialgelenks einstellen. Als einzig mögliche Therapie kann auf chirurgische Behandlung mit Meniskektomie zurückgegriffen werden (Valdez und Adams, 1978).

\section{Verletzungen der Bänder}

Das gerade Kniescheibenband ist am stärksten Verletzungen ausgesetzt. Röntgenologisch können in länger bestehenden Fällen Verdickung und verstärkte Verschattung im Band mit periostaler Aufrauhung an seiner Ansatzstelle an der Patella bestehen (Abb. 10). Vorausgesetzt, daß eine angemessene Ruhezeit gewährt wird, ist die Prognose in diesen Fällen im allgemeinen recht günstig. Ein ernsteres Problem schafft die Traumatisierung des kranialen Kreuzbandes. Ein knöcherner Ausriß proximal an der Tibia oder in der Fossa intercondylaris mit dadurch bedingter Instabilität im Gelenk ist häufiger als die vollständige Bandruptur. Auf den Röntgenaufnahmen kann ein Knochenfragment in der Fossa intercondylaris oder abgelöst vom Zwischenknorrenfortsatz der Tibia sichtbar sein. In mehr chronischen Fällen besteht eine periostale Aufrauhung und Knochenzubildung an der kranialen Ansatzstelle (Abb. 11). Die
Prognose ist von der Schwere der Bandverletzung und der Wahrscheinlichkeit der sekundären Gelenkdegeneration und Arthrosis deformans abhängig. Pferde in leichteren

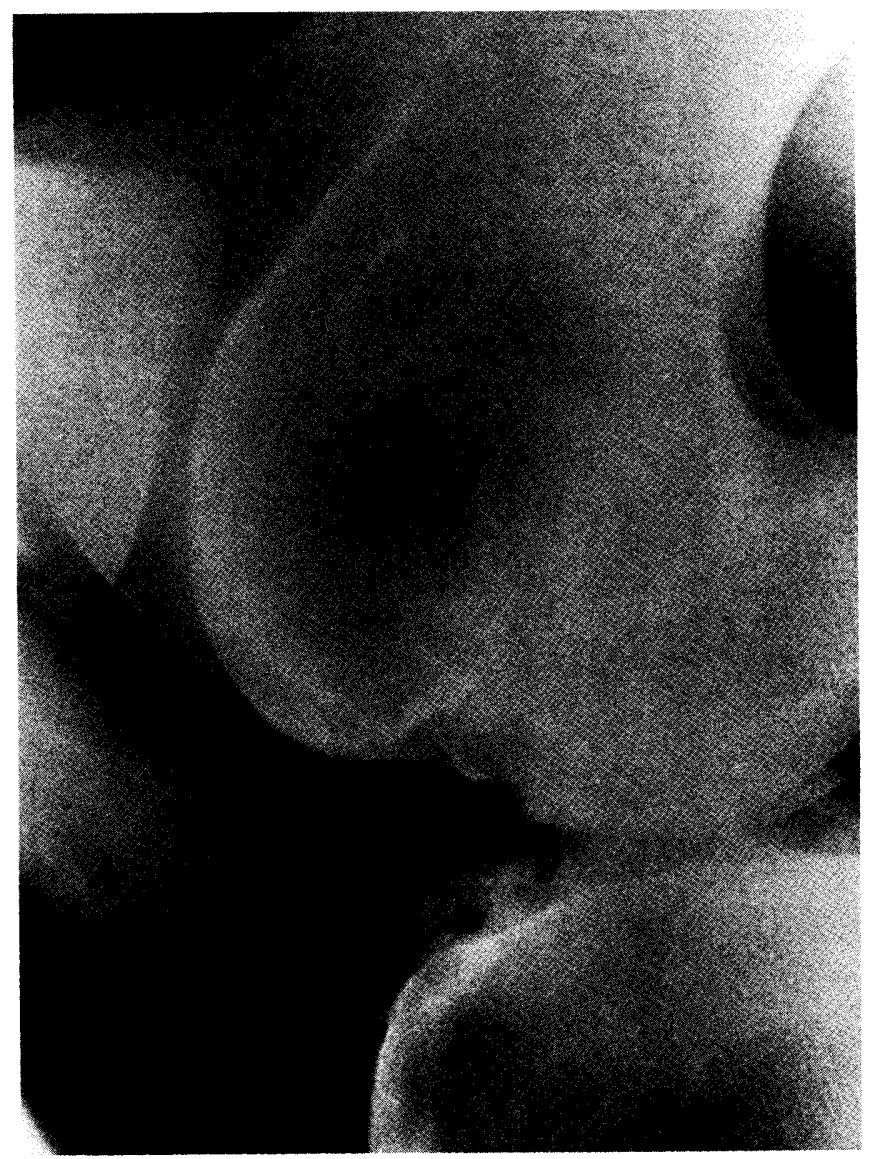

Abb. 11: Chronische Schädigung des kranialen Kreuzbands mit Knochengewebsproliferation an seiner kranialen Ansatzstelle. 
Fällen werden wieder gesund, können jedoch in der Regel keiner schweren Belastung mehr standhalten. Die Verletzung des medialen Kollateralbands des Femorotibialgelenks tritt zusammen mit Schädigungen anderen Gelenkund Weichteilgewebes auf. Anzeichen auf den Röntgenbildern dafür sind periostale Aufrauhungen an der Ansatzstelle des Bands am medialen Kondylus, im allgemeinen in Verbindung mit Veränderungen am medialen Kondylus der Tibia.

\section{Weitere mögliche Befunde}

Die laterale Luxation der Patella kommt bei erwachsenen Pferden selten vor. Sie ist in der Regel traumatisch bedingt und einseitig. Bei Ponys kann sie kongenital auftreten und ist häufig bilateral. Abgesehen von der veränderlichen Position der Patella gibt es keine sonstigen Hinweise auf die Diagnose.

Die Calcinosis circumscripta oder tumoröse Kalzinose (O'Connor und Lucey, 1977) ist ein weiterer seltener Befund am Kniegelenk des Pferdes. Es besteht eine große umschriebene schattengebende Zubildung nahe beim Kniegelenk, die es aber nicht wirklich einschließt. Die Ätiologie der Erkrankung ist unbekannt. Gewöhnlich gehen die Tiere nicht stark lahm, und die chirurgische Entfernung ist der am ehesten befriedigende Behandlungsweg.

\section{Literatur}

Röntgentechnik und röntgenanatomische Betrachtungen

Adams, O. R. (1974): Lameness in Horses, 3rd edn. Lea \& Febiger, Philadelphia.

Carlson, W. D. (1977): Veterinary Radiology, 3rd edn. Eds E. L. Gillet, D. E. Thrall und J. E. Lebel. Lea \& Febiger, Philadelphia.

Douglas, S. W., und Williamson, H. D. (1980): Principles of Veterinary Radiography, 3rd edn. Bailliere Tindall, London.

Getty, R. (1975): Sissons Anatomy of Domestic Animals, 5th edn. W. B. Saunders Co, Philadelphia.

Jeffcott, L. B. (1979): A technique for radiographic examination of the equine vertebral column. Vet. Radiol. 20, 135-139.
Jeffcott, L. B., und Kold, S. E. (1982 a): Radiographic examination of the equine stifle. Equine vet. J. 14, 25-30.

O'Brien, T. R. (1973): Radiology of the equine stifle. Proc. 19th Ann. Conv. Am. Ass. equine Pract., 271-287.

Quick, C. B., und Rendano, V. T. (1978): Equine Radiology - the stifle. Mod. vet. Pract. 59, 455-461.

Schebitz, H., und Wilkens, H. (1978): Atlas of Radiographic Anatomy of the Horse, 3rd edn. Paul Parey, Berlin.

\section{Röntgenbefunde}

Dik, K. J. (1981): Rontgenologische Aspecten van Polyarthritis bij Veulens (Radiological aspects of Polyarthritis in foals). Thesis, University of Utrecht, Netherlands.

Gerring, E. L., und Davies, J. V. (1982): Fracture of the tibial tuberosity in a polo pony. Equine vet. J. 14, 158-159.

Jeffcott, L. B., und Kold, S. E. (1982 b): Stifle lameness in the horse: A survey of 86 referred cases. Equine vet. J. 14, 31-39.

Jeffcott, L. B., und Kold, S. E. (1982 c): Clinical and radiological aspects of stifle bone cysts in the horse. Equine vet. J. 14, 40-66.

Jeffcott, L. B., Kold, S. E., und Melsen, F. (1983): Aspects of the pathology of subchondral bone cysts in the equine stifle. Equine vet. J. 15, 304-310.

Kold, S. E., und Hickman, J. (1983): Use of an autogenous cancellous bone graft in the treatment of subchondral bone cysts in the medial femoral condyle of the horse. Equine vet. J. 15, 312-316.

Moore, J. N., und McIllwraith, C. W. (1977): Osteochondrosis of the equine stifle. Vet. Rec. 100, 133-136.

O'Connor, J. P., und Lucey, M. P. (1977): Tumoral calcinosis (Calcinosis circumscripta): in the borse. Irish vet. J. 31, 173-175.

Reid, C. F. (1970): Radiographic diagnosis and appearance of osseous cystlike lesions in horses, previously reported as periarticular subchondral "bone cysts". Proc. 16th Ann. Conv. Am. Ass. equine Pract. 185-187. Rejno, S., und Strömberg B. (1978): Osteochondrosis in the horse: II Pathology. In: Osteochondrosis in Domestic Animals. Ed S. E. Olssen. Acta. Radiol. Suppl. 358.

Strömberg, B. (1979): A review of the salient features of osteochondrosis in the horse. Equine vet. J. 11, 211-214.

Strömberg, B., und Rejno, S. (1978): Osteochondrosis in the horse: A clinical and radiological investigation of osteochondritis of the knee and hock joint. In: Osteochondrosis in Domestic Animals. Ed S. E. Olssen. Acta. Radiol. Suppl. 358.

Valdez, H., und Adams, O. R. (1978): Surgical approach for medial meniscectomy in the horse. J. Am. vet. med. Ass. 165, 91-95.

Verschooten, $F$., und De Moor, A. (1982): Subchondral cystic and related lesions affecting the equine pedal bone and stifle. Equine vet. J. 14, 47-54.

\section{Leo Jeffcott, PhD, FRCVS}

Department of Veterinary Clinical Sciences

University of Melbourne

Werribee, Victoria 3030

Australia

Erschienen in EQUINE VETERINARY JOURNAL (1984): 16, 81-88, übersetzt und veröffentlicht mit freundlicher Genehmigung der British Equine Veterinary Association. 